${ }^{1}$ Hoskins, D. G., Murdoch, H. S., Hazard, C., and Jauncey, D. L., Aust. J. Phys., 25, 559 (1972).

${ }^{2}$ Hunstead, R. W., Mon. Not. Roy. Astron. Soc., 152, 277 (1971).

3 Adgie, R. L., Crowther, J. H., and Gent, H., Mon. Not. Roy. Astron. Soc., 159, 233 (1972).

${ }^{4}$ Gower, J. F. R., Scott, P. F., and Wills, D., Mem. Roy, Astron. Soc., 71, 49 (1967).

5 Kellermann, K. I., Jauncey, D. L., Cohen, M. H., Shaffer, B. B. Clark, B. G., Broderick, J., Rönnäng, B., Rydbeck, O. E. H., Matveyenko, L., Moiseyev, I., Vitkevitch, V. V., Cooper, B. F. C., and Batchelor, R., Astrophys. J., 169, 1 (1971).

6 Blake, G. M., Astrophys. Lett., 6, 201 (1970).

7 Fanaroff, B. L., and Blake, G. M., Mon. Not. Roy. Astron. Soc., 157, 41 (1972).

8 Burbidge, E. M., and Strittmatter, P. A., Astrophys. J. Lett., 174, L57 (1972).

9 Véron, M. V., Astron. Astrophys., 11, 1 (1971).

10 Véron, M. V., Astron. Astrophys., 20, 471 (1972).

\section{Relativistic Behaviour of Circumnavigating Clocks}

CONSIDERABLE interest has been aroused by Hafele's suggestion $^{1,2}$, subsequently supported by experiment ${ }^{3}$, that caesium beam clocks, borne by commercial jets circumnavigating the Earth, may provide supporting evidence for the famous prediction of Einstein's theory of relativity that if one member of a set of twins departs from the Earth in a spacecraft and later returns to reunite with his sibling, he will be the younger of the two. Strictly speaking, this statement is valid only if the frame of reference in which the home-bound sibling remains at rest is an inertial frame, which is not true of a frame of reference attached to the (rotating) Earth. This fact was ordinarily disregarded because the relative velocities involved were supposed to be much larger than the velocity difference caused by this circumstance. But in the case of clocks employed by Hafele and Keating ${ }^{3}$, this supposition is not true and the influence of Earth's rotation must be reckoned with.

In Hafele's calculation ${ }^{1,2}$, a nonrotating frame of reference embedded in the Schwarzschild metric of the Earth played an intermediate role. Of course, the final result was expressed in terms of the ground speed of the flying clock. For flights confined to circular orbits in the equatorial plane, Hafele showed that

$$
\left(\mathrm{d} \tau / \mathrm{d} \tau_{0}\right) \simeq 1+g h / c^{2}-\left(2 \Omega R u+u^{2}\right) / 2 c^{2}
$$

where $g=G M / R^{2}-\Omega^{2} R$ and $M, R$ and $\Omega$ are the mass, the radius and the angular velocity of the Earth. Equation (1) contains a directional effect which arises from the term linear in $u$ ( $u$ positive for eastward flights, negative for westward) and is related to the fact that the speed $u$ is measured in a rotating frame of reference. This seems to have been overlooked by Schlegel ${ }^{4}$ when he suggested that the directional effect appearing in equation (1) may be erroneous. Here, I present a calculation, made directly in the rotating frame of reference, which not only vindicates Hafele's result but also generalizes it to more practical situations.

In an arbitrary frame of reference, the space-time metric is

$$
\mathrm{d} s^{2}=g_{i k} \mathrm{~d} x^{i} \mathrm{~d} x^{k}
$$

where $x^{i} \equiv\left(x^{\alpha}, c t\right) ; \alpha=1,2,3$. The metric tensor $g_{t k}$ includes the effects of gravitation as well as of the noninertial nature of the frame of reference. The proper time of a standard clock is then given $b y^{5}$

$$
\mathrm{d} \tau=\mathrm{d} t\left[-g_{44}-2 g_{\alpha 4}\left(u^{\alpha} / c\right)-g_{\alpha \beta}\left(u^{\alpha} u^{\beta} / c^{2}\right)\right]^{1 / 2}
$$

where $u^{\alpha}=\left(\mathrm{d} x^{\alpha} / \mathrm{d} t\right)$. For a clock at rest, equation (3) reduces to

$$
\mathrm{d} \tau=\mathrm{d} t\left(-g_{44}\right)^{1 / 2} \simeq \mathrm{d} t\left(1+\Phi / c^{2}\right)
$$

where $\Phi$ is the Newtonian potential at the position of the clock. In a Lorentz frame, this becomes

$$
\mathrm{d} \tau=\mathrm{d} t\left(1-u^{2} / c^{2}\right)^{1 / 2}
$$

Now, if the underlying metric is of the Schwarzschild type (arising from the mass $M$ of the Earth) and is viewed from a frame of reference $K$ which rotates, with angular velocity $\Omega$, with respect to the system of "fixed" stars, then the nonvanishing elements of the metric tensor, in the spherical coordinates $(r, \theta, \varphi)$, are given by ${ }^{6}$

$\left.\begin{array}{l}g_{11}=\left(1-\frac{2 G M}{c^{2} r}\right)^{-1}, g_{22}=r^{2}, g_{33}=r^{2} \sin ^{2} \theta \\ g_{44}=\left(1-\frac{2 G M}{c^{2} r}-\frac{\Omega^{2} r^{2} \sin ^{2} \theta}{c^{2}}\right), g_{34}=g_{43}=\frac{\Omega r^{2} \sin ^{2} \theta}{c}\end{array}\right\}(4)$

Substituting (4) into (3), and for slow motions $\left(u^{\alpha} \ll c\right)$ and weak fields $\left(\Phi \ll c^{2}\right)$,

$$
\begin{aligned}
& \mathrm{d} \tau \simeq \mathrm{d} t\left[1-\left(\frac{G M}{c^{2} r}+\frac{\Omega^{2} r^{2} \sin ^{2} \theta}{2 c^{2}}\right)-\right. \\
&\left.\left(\frac{2 \Omega r^{2} \sin ^{2} \theta(\mathrm{d} \varphi / \mathrm{d} t)+u^{2}}{2 c^{2}}\right)\right]
\end{aligned}
$$

In comparing the rates of two given clocks, the coordinate differential $\mathrm{d} t$ is eliminated.

Equation (5) contains two effects: the effect of altitude which, for $r=R+h(h \ll R)$, takes the form $g h / c^{2}$ where $g\left\{=G M / R^{2}-\Omega^{2} R \sin ^{2} \theta\right\}$ is the "measured" value of the acceleration due to gravity at a place of colatitude $\theta$; and the effect of motion of the clock which is directional, because of the occurrence of the product $\Omega(\mathrm{d} \varphi / \mathrm{d} t)$, and for $\theta=90^{\circ}$ reduces to the Hafele expression $-\left(2 \Omega R u+u^{2}\right) / 2 c^{2}$.

Equation (5) may be applied to a satellite-borne clock as well. I consider motion in a circular orbit, of radius $R^{*}$, inclined at an angle $\lambda$ to the equatorial plane of the Earth. Measuring $\varphi$ from the line of nodes, in frame $\mathrm{K}$

$$
\theta=\cos ^{-1}(\sin \lambda \sin \alpha), \varphi=\tan ^{-1}(\cos \lambda \tan \alpha)-\Omega t
$$

where angle $\alpha$ locates the satellite in its own plane of rotation: $\dot{\alpha} \equiv \omega=\sqrt{G M / R^{*}}$. Equation (5) then gives

$$
\mathrm{d} \tau^{*} \simeq \mathrm{d} t\left(1-3 G M / 2 c^{2} R^{*}\right)
$$

which is independent of $\lambda$ and $\Omega$, and depends only on $R^{*}$. In $\mathrm{K}_{0}$ one would have

$$
\mathrm{d} \tau * \simeq \mathrm{d} t\left(1+\Phi / c^{2}-u^{2} / 2 c^{2}\right)
$$

which, for $\Phi=-G M / R^{*}$ and $u^{2}=\omega^{2} R^{2}=G M / R^{*}$, would again lead to equation (6).

Finally, comparing the satellite-borne clock with an Earthbound clock (for which $\Phi=-G M / R$ and $u=0$ ),

$$
\left(\mathrm{d} \tau * / \mathrm{d} \tau_{\mathrm{E}}\right) \simeq 1-\left(G M / 2 c^{2}\right)(3 / R *-2 / R)
$$

the $\Omega$-term is now clearly negligible. Effects predicted by equation (7) are about 100 times larger than the ones encountered in the case of jet-borne clocks. Establishment of Skylab may subject these effects to a more decisive test.

This work was partly supported by the National Research Council of Canada.

\section{R. K. Pathria}

Department of Physics,

University of Waterloo,

Waterloo, Ontario

${ }^{1}$ Hafele, J. C., Nature, 227, 270 (1970).

2 Hafele, J. C., Amer. J. Phys., 40, 81 (1972).

3 Hafele, J. C., and Keating, R. E., Science, 177, 166 (1972).

${ }^{4}$ Schlegel, R., Nature Physical Science, 229, 237 (1971).

5 Pathria, R. K., The Theory of Relativity, Sec. 8.7 (Hindustan Publishing Corporation, Delhi, 1963).

${ }^{6}$ Møller, C., The Theory of Relativity, Sec, 90 (Oxford Univ. Press, 1952). 